\title{
Chemotherapy for brain tumors of astrocytic and oligodendroglial lineage: The past decade and where we are heading ${ }^{1}$
}

\author{
Victor A. Levin ${ }^{2}$ \\ Department of Neuro-Oncology and the Brain Tumor Center, M.D. Anderson Cancer Center, University of \\ Texas, Houston, TX 77030
}

Over the past three decades, we have made great strides in the treatment of most, but not all, brain tumors. Dramatic advances have occurred in diagnostic imaging, neurosurgery, neuroanesthesia, radiotherapy, and chemotherapy for CNS tumors. Unfortunately, our progress has not yet met our expectations. Because of the infiltrative nature of most primary brain tumors, neurosurgery can never be expected to be curative for the majority of gliomas. Because infiltrative tumors interdigitate with normal brain cells and are not highly sensitive to irradiation, one cannot expect radiotherapy to be curative without serious damage to normal brain cells. The hope for a cure, then, rests with chemotherapy. Those who administer chemotherapy to patients with CNS tumors fully expect that, in time, long-term survival and, ultimately, the cure will become an everyday reality. To achieve that reality, however, new treatment concepts and drugs are needed. Neuro-Oncology 1, 69-80, 1999 (Posted to Neuro-Oncology [serial online], Doc. 98-08, January 19, 1999. URL <neuro-oncology.mc.duke.edu>)

Received 14 July 1998, accepted 24 September 1998.

${ }^{1}$ This work was partially supported by National Cancer Institute Grant PO1 CA55261.

${ }^{2}$ Address correspondence and reprint requests to Victor A. Levin, MD, Department of Neuro-Oncology-100, M.D. Anderson Cancer Center, University of Texas, 1515 Holcombe Boulevard, Houston, TX 77030.

${ }^{3}$ Abbreviations used are as follows: $A A$, anaplastic astrocytoma; $A G$, anaplastic glioma; AGAT, $\mathrm{O}^{6}$-alkylguanine-DNA alkyltransferase; $A O$, anaplastic oligodendroglioma; $\mathrm{BrdU}$, bromodeoxyuridine; $\mathrm{Cl}$, confidence interval; GBM, glioblastoma multiforme; IrdU, iododeoxyuridine; NCOG, Northern California Oncology Group; OA, oligoastrocytoma; PCNU, 1-(2-chloroethyl)-3-(2,6-dioxo)-3-piperidyl1-nitrosourea; PCV, procarbazine, lomustine, vincristine; RTOG,

Radiation Therapy Oncology Group; TTP, time to tumor progression.
W hat do we consider as cancer chemotherapy today? Our dictionaries define "drug" as a substance used as a medicine. "Medicine" is defined as a substance or preparation used in treating disease. We can all agree that phenytoin and penicillin are drugs by those definitions. As we approach the 21st century, however, our perception of what an anticancer drug is will probably change. When we talk about anticancer chemotherapy, we think mostly of cytotoxic drugs such as carmustine, lomustine, and procarbazine. During the past 10 years, our colleagues in the oncology community coined a new term for the interferons and neutrophil-activating proteins, calling them "biological response modifiers." In fact, the interferons are substances used to treat disease and, as such, should rightfully be called drugs. Will substances that interfere with angiogenesis or tumor invasion be considered drugs? Will naming depend on whether the body normally produces these proteins or they are created by the pharmaceutical industry? Will introduction of gene products using adenoviral vectors continue to be called "gene therapy" if liposomal vehicles used to deliver gene products are referred to as drugs? For the sake of simplifying this review, I use the term "chemotherapy" to encompass cytotoxic drugs and direct-acting biological response modifiers, as well as any substance that can be administered by mouth, injection, or direct contact and exerts an effect on the biological behavior of cancer cells (tumor growth) in the host.

\section{The Cytotoxic Era: Systemic Drugs}

The era of cytotoxic chemotherapy for brain tumors began in the mid-1960s with the organization of the Brain Tumor Study Group and their initial trials of surgery, irradiation, and carmustine. They demon- 
strated a modest improvement in survival for $\mathrm{GBM}^{3}$ patients who were treated with carmustine in combination with radiation and surgery compared with those treated only with radiation and surgery. This led, in the early 1970s, to Food and Drug Administration approval of carmustine and lomustine for the treatment of brain tumors. Over the next 25 years, neurosurgeons, neurologists, adult and pediatric medical oncologists, and radiation oncologists in the United States, Europe, and Japan conducted clinical trials of cytotoxic drugs alone and in combination for the treatment of gliomas and medulloblastomas.

During this era, advances were made in the treatment of low-grade astrocytoma, AA, oligodendroglioma, AO, and medulloblastoma. For GBM, however, little appreciable gain in survival has been achieved since the early 1970 s. Most of us who consider ourselves neuro-oncologists attribute much of the improvement in survival to chemotherapy; however, improvements in neuroimaging, neurosurgery, and radiotherapy have also contributed to the increases in survival for children and adults with primary CNS tumors. On the basis of a career in neuro-oncology that spans more than 30 years, I hold the bias that some forms of chemotherapy add more than others to patients' relapse-free survival, overall survival, and quality of life.

\section{Adjuvant Pre-irradiation Chemotherapy for High-grade Gliomas}

Pre-irradiation chemotherapy has been reported in the neuro-oncology literature for nearly 15 years. A recent study of 37 adults with high-grade gliomas suggested that survival for GBM patients might not be decreased if radiotherapy is delayed 8 weeks (Kirby et al., 1996). This finding supports the feasibility of evaluating chemotherapy regimens before radiotherapy in patients with GBM. A study from Italy of patients with AG treated with 4-5 cycles of carmustine followed by radiation and then postirradiation procarbazine, lomustine, and vincristine found a median survival of 3.2 years (Silvani et al., 1995), a result that is actually better than those reported for studies of post-radiotherapy carmustine (Levin et al., 1990).

Grossman et al. (1997) reported a phase II study of 52 adults who were treated with 72-hour continuous infusions of carmustine and cisplatin every 3-4 weeks for 3 cycles, which were followed by conventional external beam irradiation. The reported median survival of 13 months, given the distribution of GBM to AA (from 88 to $12 \%$ ), indicates that this treatment regimen is no more active than many others used to date to treat high-grade gliomas (Levin et al., 1997).

The situation can be a bit more complex in children than in adults because of the higher incidence of neuraxis dissemination in children. In adults, the incidence of neuraxis dissemination is typically less than $5 \%$ (Choucair et al., 1986); in children, it can be more than $25 \%$ (Heideman et al., 1997). In a study by Heideman et al. (1997), 41 children with newly diagnosed supratentorial malignant gliomas (13 AA, $25 \mathrm{GBM}$, and 3 $\mathrm{AO})$ were treated with pre-irradiation chemotherapy between 1984 and 1994. The investigators found that children who had gross total resection survived longer than those who underwent subtotal resection or biopsy $(P=0.004)$, but the survival benefit from neoadjuvant chemotherapy studies was not significantly different from that from adjuvant chemotherapy studies.

Summary. Although great interest in pre-irradiation chemotherapy has surfaced in the past decade, virtually no evidence exists that, with the cytotoxic regimens evaluated to date, neoadjuvant chemotherapy produces longer survival than adjuvant chemotherapy for children or adults with supratentorial malignant gliomas. Because neoadjuvant chemotherapy does not appear to reduce efficacy, some investigators evaluate new therapies in this interval before irradiation. The difficulty with this approach is that if response is the endpoint, investigators must deal with postsurgical radiographic artifacts that can contaminate MRI response criteria. If durability of response is the endpoint, then treatment must proceed a sufficiently long time and irradiation must be withheld (for some time at least). If the preirradiation chemotherapy is outstanding, this study design presents no problem; if not, waiting may require a second operation before irradiation if rapid tumor growth occurs in the interim. With these limitations in mind and because the eradication of radiotherapy is a legitimate goal of chemotherapy, this approach makes sense for future generations of anticancer therapies.

\section{Adjuvant Chemotherapy During Radiotherapy for High-grade Gliomas}

The term "chemoradiation" refers to the use of drugs to enhance tumor cell kill during radiotherapy and thereby increasing patient survival. Chemoradiation has been widely evaluated for cancers throughout the body. Historically, approaches have ranged from the use of agents to circumvent the problem of hypoxia in the tumor environment to the use of cytotoxic drugs that potentiate radiation-induced DNA damage and reduce the likelihood of DNA repair. To date, neither approach has led to significant survival gains for patients with CNS tumors, even when accelerated hyperfractionation has been used to shorten radiotherapy, allowing for the administration of higher drug doses.

In 1983, my colleagues and I conducted a phase II study to evaluate the long-term efficacy and safety of radiotherapy combined with intravenous BrdU for patients with GBM and AG (Levin et al., 1995b; Phillips et al., 1991). Patients received a BrdU infusion of $0.8 \mathrm{~g} \cdot \mathrm{m}^{-2} \cdot 24 \mathrm{~h}^{-1}$ for 4 days during each of the 6 weeks of radiotherapy $(60 \mathrm{~Gy})$; this was followed by chemotherapy with PCV for 1 year or until progression. Median survival for the 160 evaluable patients with GBM was 55.7 weeks (Phillips et al., 1991). A multivariate analysis indicated that age, Karnofsky performance status, and BrdU dose were significant predictors of time to failure; age and Karnofsky performance status were significant predictors for survival. The 116 evaluable patients with AG did better, that is, the estimated 4-year survival rate was $46 \%$. For patients with low-grade infiltrative astrocytoma $(n=22)$, the estimated 6-year survival rate was $79 \%$. Whole-brain irra- 
diation was given to the first $23 \%$ of patients and limited-field irradiation was given to the remaining $77 \%$; patients receiving limited-field irradiation had a better survival rate $(P=0.07)$. As a result of this study, Prados and colleagues conducted a randomized study of concurrent BrdU and radiotherapy versus no BrdU during radiotherapy; both groups received postirradiation chemotherapy with PCV. The study is closed and a final report will appear in 1999 (M.D. Prados, personal communication, 1998).

While awaiting the results of the randomized study, however, Prados et al. (1998a) compared data from the NCOG studies of BrdU chemoradiation with that from the historical RTOG studies of GBM and AA. Data from $89 \%$ (296 of 334) of patients treated with BrdU in NCOG Study 6G-82-1 and 85\% (1478 of 1743) of patients treated without BrdU in three RTOG studies were analyzed. The median survival of patients with GBM was 9.8 months in the RTOG studies and 13.0 months in the NCOG trial $(P<0.0001)$. For patients with AA, the median survival was 35.1 months in the RTOG studies and 42.8 months in the NCOG trial $(P=0.126)$. A proportional hazards regression model, which included treatment type, pathologic classification, Karnofsky performance status, age, and extent of surgery, demonstrated that treatment with BrdU was included in the best model for only patients with GBM (risk ratio 0.83).

To maximize the drug dose during irradiation and enhance the chemoradiation effect, my group conducted a phase II single-arm study of accelerated fractionated radiotherapy combined with intravenous carboplatin for patients with previously untreated GBM (Levin et al., 1995a). Eighty-three adults received 1.9-2.0 Gy radiation 3 times a day with 2-h infusions of $33 \mathrm{mg} / \mathrm{m}^{2}$ carboplatin before each radiation dose for 25 -day cycles separated by 2 weeks; following radiotherapy, patients received PCV for 1 year or until tumor progression. Seventy-four patients $(89 \%)$ received one or more courses of PCV; their median survival was 55 weeks. A multivariate analysis showed that age $(P=0.013)$ and extent of initial surgery $(P=0.003)$ together were predictive of a better survival; no other variables provided additional statistical significance. Only $8.4 \%$ of patients had clinically documented therapy-associated CNS side effects (radiation necrosis). Survival in this study is similar to that currently attainable with other chemoradiation approaches. We have recently completed, but not yet reported, a single-arm phase II study of 250 patients with GBM using the same radiotherapy schedule with high-dose $\operatorname{BrdU}\left(8.4 \mathrm{~g} \cdot \mathrm{m}^{-2}\right.$. $24 \mathrm{~h}^{-1} \times 4$ days) for each of the 2 weeks.

In a recent small study from Europe, Brandes et al. (1998) treated 56 patients with GBM with conventional limited-field radiotherapy (60 Gy) and concurrent chemotherapy consisting of 3 days of carboplatin and 9 days of teniposide during 6 weeks of radiotherapy. After irradiation, patients were given carmustine every 8 weeks for 3 cycles. The median survival time was 12.5 months, which is comparable to results reported for radiotherapy alone followed by chemotherapy with nitrosoureas. Note that four patients $(7.1 \%)$ had radia- tion necrosis at 2, 2, 7, and 9 months from the end of radiotherapy.

Summary. Unfortunately, none of the chemoradiation trials to date have unequivocally demonstrated a therapeutic gain for patients with supratentorial malignant gliomas. The benefits of halopyrimidines (BrdU, IrdU) for GBM are small at best. In addition, irradiation of the CNS produces evidence of damage seen on MRI in $\sim 10-35 \%$ of patients, depending on radiation dose and fraction size (Corn et al., 1994; Nishimura et al., 1992; Van Tassel et al., 1995). There is also concern that chemoradiation increases neurologic side effects. The minority view suggests that brain irradiation to 50 or 64 Gy (Hammack et al., 1995) and hyperfractionation to 80 Gy (Jason et al., 1997) does not produce neurocognitive impairment. The majority view suggests that brain irradiation produces high levels of impairment in memory, executive function, sustained attention, motor processing, and information processing (Scheibel et al., 1996). While logic supports the expectation that adding chemotherapy to radiotherapy may actually increase toxicity, few studies actually address this concern (Postma et al., 1998). Nonetheless, given present knowledge and experience, pursuing chemoradiation is a counterintuitive strategy.

\section{Adjuvant Postirradiation Chemotherapy for High-grade Gliomas}

Adjuvant postirradiation chemotherapy for GBM has evolved little over the past decade; however, there is greater support for combination chemotherapy after radiotherapy for AGs. With several different regimens, the median survival for patients with AG is 3-5 years, and as many as $40 \%$ of patients are alive at $8-10$ years (Levin et al., 1995b; Prados et al., 1998a, 1998b). Unfortunately, a review of phase III and smaller phase II trials of postirradiation chemotherapy indicates that we are at a plateau with respect to patient survival for both GBM and AA.

In both children and adults, the 8-drugs-in-1-day combination did not prove to be better than less toxic and simpler therapies (Finlay et al., 1995). In a randomized trial of postirradiation chemotherapy in 192 children between 1.5 and 21 years of age with highgrade astrocytomas, the 8-drugs-in-1-day chemotherapy regimen was not superior to lomustine, vincristine, and prednisone.

Knerich et al. (1990) reported on a large randomized trial with chemotherapy, which was begun at the same time as radiotherapy and continued after radiotherapy was completed. A total of 173 patients with malignant glioma were randomly assigned to receive lomustine alone, lomustine plus teniposide, or lomustine, teniposide, and 5-fluorouracil. Statistical analysis of 150 evaluable patients showed a better quality of life and survival for patients treated with the 3-drug combination than for those treated with the 2-drug or single-drug therapy (median survival for the 3 groups was 18.2 months, 14.7 months, and 13.8 months, respectively; $P<0.01$ ), but also a higher incidence of CNS side effects.

Data from NCOG protocol 6G61, which closed in February 1983, were reanalyzed in December 1988 
(Levin et al., 1990). Patients in the trial were randomly assigned to receive 60 Gy external beam irradiation with oral hydroxyurea, followed by either carmustine or PCV. The PCV combination produced longer survival and TTP than carmustine for both GBM and AG, although the difference was statistically significant only for the patients with AG. In patients with AG, TTP and median survival was twice as long after PCV treatment (146 vs. 82 weeks).

In search of new chemotypes, the National Cancer Institute made available diaziquone and carmustine. In the first study, diaziquone was evaluated against carmustine in a randomized study of 251 patients with AG who had been treated with surgery and radiotherapy (Schold et al., 1993). There was no significant difference in either TTP or survival between the two treatment arms. The investigators found that older patients ( $>45$ years old) with glioblastoma or gliosarcoma had a median survival of 37 weeks after randomization, and younger patients ( $<45$ years old) with AG (mostly AA) had a median survival of 147 weeks. For patients with AG, there was no significant difference in efficacy between diaziquone and carmustine, although diaziquone was somewhat better tolerated. Patients randomly assigned to diaziquone who had significant hematologic toxicity that required dose reduction after the first treatment cycle, however, had significantly longer TTP and survival than did those who did not require dose reduction $(P=0.011$ and 0.016 , respectively). Patients requiring dose reduction had greater myelotoxicity, which may also imply that these patients had a genetic abnormality (advantage) that also enhanced the antitumor efficacy of diaziquone. It is conceivable that these patients may have had a longer plasma half-life (higher area under the curve) or possibly a reduced capacity to repair diaziquone-induced DNA damage.

In another study, the Brain Tumor Study Group randomly assigned 152 adults with primary brain tumors to receive PCNU every 8 weeks or diaziquone once a week for 4 weeks every 6 to 8 weeks (Malkin et al., 1994). Median survival times were 11.0 months for the PCNU group and 8.4 months for the diaziquone group. Life-table analysis estimates for 2-year survival rates were $34 \%$ for patients treated with PCNU and $11 \%$ for diaziquone.

Recently, Prados et al. (1998b) reported a treatment for malignant gliomas consisting of radiotherapy concomitant with hydroxyurea followed by chemotherapy with carmustine and 6-thioguanine. Their study included 135 patients with GBM and 110 patients with AG (103 with AA, 7 with high-grade OA). For patients with GBM, the median survival was 56 weeks $(95 \%$ CI $49,69)$. For the AG group, the median survival was not reached (95\% lower confidence bound, 284 weeks), with a median follow-up for surviving patients of 298 weeks. A proportional hazards model showed that a higher Karnofsky performance score and a lower age predicted longer survival $(P<0.01$ and $P<0.001$, respectively) in patients with GBM, and that a younger age predicted longer survival $(P=0.05)$ for patients with AG. The survival of patients with AG in this study is comparable to the survival of similar patients in recent studies using halogenated pyrimidines during radiotherapy and PCV as adjuvant chemotherapy.

A number of smaller postirradiation chemotherapy studies were also conducted, but none had sufficient statistical power or promising results to encourage further study. Hellman et al. (1998) reported a median survival of 14 months in a phase II trial with chemotherapy with a combination of etoposide, vincristine, and procarbazine in 27 patients having GBM and 6 having AA. In a phase II study, Boiardi et al. (1992) randomly assigned 84 patients with GBM to carmustine alone, carboplatin and etoposide, carboplatin and carmustine, or carboplatin, carmustine, and etoposide, and found that survival did not differ among the four therapies. In another phase II study, 38 patients with World Health Organization grade III and IV gliomas were treated after surgery and radiotherapy with a combination of carboplatin and oral tamoxifen at doses ranging from 40 to $120 \mathrm{mg} /$ day (Mastronardi et al., 1998). Median survival was 13 months. Given a mixture of histologic types in this study, this result does not suggest unusual antitumor activity of the combination. In another phase II study, 17 patients with GBM, 14 patients with $\mathrm{AA}$, and 2 patients with $\mathrm{AO}$ were treated after surgical resection with alternating intravenous carmustine and cisplatin both during and after radiotherapy (Yung et al., 1992). Although 52\% (17 of 33) patients were alive at 18 months (6 GBM, 9 AA, $2 \mathrm{AO}$ ), the results did not support a larger study.

Summary. Postirradiation chemotherapy is the area of greatest need and hope for the future. It is disappointing that progress over the past decade has not been greater. Although postirradiation chemotherapy for GBM benefits few patients, those with AA (as well as $\mathrm{AO}$ and $\mathrm{OA}$ ) benefit more from some drug combinations than others. Unfortunately, we have not yet progressed from nitrosourea combinations to more effective combinations.

\section{Overcoming Resistance to Nitrosoureas}

The problem of resistance to chemotherapy has been hotly pursued in the cancer biology literature and in clinical trials over the past decade. The premise of this work posits that current cytotoxic anticancer drugs would be more effective if we understood how to reduce or prevent enzymatic cellular mechanisms that inactivate the drugs at the tumor cell without inactivating anticancer drugs in dose-limiting normal cells. While this objective was readily achieved in cultured cells and some animal models, it has been an elusive objective in cancer patients. Following the administration of a nitrosourea, a monofunctional adduct attached to guanine can be removed by the enzyme AGAT. In high-grade gliomas, one mechanism of resistance to nitrosoureas is a high AGAT level. For instance, $30 \%$ of high-grade glioma patients have low levels of AGAT mRNA, and these patients have higher partial response rates to carmustine than those with high AGAT levels (Mineura et al., 1994). In a retrospective study of 167 patients treated with carmustine, survival was inversely related to AGAT levels (Mason et 
al., 1997). Unfortunately, the use of commercially available drugs such as procarbazine and dacarbazine, which can block AGAT activity and thus enhance nitrosourea activity, have failed to produce significant improvement in outcome for patients with high-grade gliomas (Ikeda et al., 1996; Kyritsis et al., 1996; Levin and Prados, 1992). A better inhibitor of AGAT, O6benzylguanine, is under investigation in combination with nitrosoureas for high-grade gliomas. To date, cell culture and animal studies indicate that blocking tumor AGAT with O6-benzylguanine can increase nitrosourea cytotoxicity (Dolan et al., 1991, 1994; Schold et al., 1996). Unfortunately, this treatment also results in an increase in systemic toxic effects in rodents unless the O6-benzylguanine is given by the intra-arterial route to increase levels of the drug in tumors and reduce systemic organ exposure (Kurpad et al., 1997).

Summary. Strategies to date that were designed to overcome resistance to alkylating agents such as the nitrosoureas have met with little success. Most efforts have focused on pretreatment with procarbazine and dacarbazine. While there is an expectation that O6-benzylguanine will be significantly more effective in blocking AGAT, it is problematic whether O6-benzylguanine enhancement of nitrosourea antitumor efficacy will incrementally improve survival for patients with glial tumors without increasing the systemic toxicity of nitrosoureas.

\section{Intra-arterial Chemotherapy}

For the past 20 years, intra-arterial chemotherapy has been pursued with the goal of improving drug delivery to brain tumors. Unfortunately, despite many theoretical advantages, these studies have produced no conclusive evidence of benefit. Most studies have used a nitrosourea, a platinoid, or both (Chauveinc et al., 1996; Iwadate et al., 1995). An example of a recent trial is a small study of 38 patients treated with nimustine and cisplatin in which a response rate of $59 \%$ (19 of 32) was observed (Iwadate et al., 1995). Local recurrence was observed in $53 \%$ of patients and recurrence more than $3 \mathrm{~cm}$ from the original tumor margin was observed in $39 \%$. The median survival time was 59 weeks for patients without distant recurrence and 42 weeks for patients with distant recurrence (not statistically significant). In the few randomized studies of intra-arterial versus intravenous chemotherapy, clear benefit of intraarterial therapy was not shown and neurologic and optic system side effects tended to detract from patient quality of life (Hiesiger et al., 1995).

To overcome CNS toxicity, Dropcho et al. (1998) evaluated selective intra-arterial cisplatin infused into either the cervical internal carotid artery (15 patients) or 1-2 major cerebral arteries (26 patients). The median TTP among the 13 (32\%) patients with tumor response or stable disease was 24 weeks. The response rate did not significantly differ between the two groups and, although selective intracerebral artery cisplatin infusion reduced the risk of optic system side effects, it did not produce a better tumor response rate, but was associated with a higher risk of seizures and neurologic side effects than was internal carotid artery infusion.
Summary. Many different approaches to intra-arterial infusion and catheter placement have been tried over the years. Intra-arterial therapy is ineffective with the agents available, however, and its cost-benefit ratio is not good enough to justify its continued pursuit with today's cytotoxic drugs.

\section{High-dose chemotherapy}

The use of high-dose chemotherapy followed by bone marrow or stem cell transplantation to increase chemotherapy dose toleration, and thereby improve survival, in brain tumor patients remains controversial. This strategy has been pursued in children and young adults far more vigorously than in older adults, but still with variable results (Dunkel et al., 1996; FernandezHidalgo et al., 1996). While response to treatment has been observed and treatment morbidity has declined with experience, true therapeutic advantage has not been proven (Finlay, 1996; Finlay et al., 1990, 1996).

Summary. Ideally, it would be valuable to conduct randomized trials of high-dose versus conventional dose chemotherapy in glioma patients. Unfortunately, many problems are inherent in controlled clinical trials of these treatments: (1) in the younger age group, there are limited numbers of patients; (2) parents who want to do everything for their children will be unwilling to forgo the promise (and the hype) of transplantation to let their children be part of a randomized study; (3) insurance coverage of these expensive procedures will be hard to arrange in a randomized study; and (4) agreement on appropriate drugs to be used may be problematic. When reviewing published studies, recall that treatment with radiation and chemotherapy invariably produces better survival in patients between $\sim 12$ and 45 years of age than in patients of other ages, a phenomenon that confounds results reported in the literature. Typical drugs used have been carmustine, thiotepa, cisplatin, and etoposide. None of these drugs have antitumor activity at conventional doses, and, in fact, thiotepa is nearly without activity against gliomas at lower doses than those used for transplantation.

\section{The Special Case of Chemotherapy for Oligodendrogliomas}

Of the glial neoplasms, those of oligodendroglial lineage appear easiest to control from a surgical, radiotherapeutic, and chemotherapeutic standpoint. On average, patients with low-grade tumors survive 9 years, whereas those with high-grade tumors survive 2.2 years (Berger et al., 1995; Levin et al., 1997). For the most part, patients reported in those series were treated with surgery and radiation; few were treated with chemotherapy as part of the clinical trial.

Interestingly, oligodendroglioma tumor cells can cohabitate with cells that are phenotypically malignant astrocytes. Tumors with both kinds of cells are called oligoastrocytomas and can be low grade or anaplastic. BrdU-labeling index studies in 47 patients with oligoastrocytomas of various degrees of malignancy found $70 \%$ of tumors had low-grade astrocytoma components, $28 \%$ had anaplastic astrocytoma components, and $74 \%$ had oligodendroglioma grade B components 
(Wacker et al., 1994). For a BrdU-labeling index $>5 \%$, median TTP was 4.5 months, but for tumors with a labeling index $<5 \%$, the median TTP was not reached. Although investigators concluded that survival correlates with the BrdU-labeling index of the oligodendroglial component, this conclusion is paradoxical and not well supported. Most of the tumors consisting of oligodendroglioma grade B with AA had higher labeling indices $(>2.5 \%)$, and most of the tumors consisting of oligodendroglioma with low-grade astrocytoma had lower labeling indices $(<1 \%)$. In addition, clinical experience supports the observation that progressive oligoastrocytomas eventually develop the histological appearance of GBM or AA; they do not, as a rule, develop the phenotypically distinct appearance called oligodendroglioma grade D.

For the most part, oligodendroglioma is managed with wide surgical resection and radiotherapy for residual tumor. The case for AO is more problematic. Cairncross et al. (1994) have conducted a series of consecutive phase II studies to examine the rate and duration of response of AO to PCV. In a study of 24 eligible patients, they found that $38 \%$ had a complete response, $38 \%$ had a partial response, and $17 \%$ had stable disease. The median TTP was $>25$ months for complete responders, 14 months for partial responders, and 6.8 months for patients with stable disease.

Kyritsis et al. (1993) reviewed a series of 8 patients with $\mathrm{AO}$ initially treated with radiotherapy and adjuvant chemotherapy and 12 patients with AO treated at the time of recurrence (includes 3 who failed adjuvant chemotherapy) with salvage chemotherapy. Of the 8 patients who received adjuvant chemotherapy, all had a response or stable disease and 7 had a relapse by 10 months. Of the 12 patients treated at recurrence, $3 \mathrm{had}$ a response and 6 had stable disease; survival times ranged from 15 to greater than 132 months (Allison et al., 1997). This small review suggests that, while AO is a relatively chemotherapy-sensitive glioma, adjuvant chemotherapy after irradiation may have a minimal advantage over a wide surgical resection followed by conventional radiotherapy for the initial treatment of AO.

Allison et al. (1997) reported on a series of 14 patients with oligodendroglioma, 3 with AO, and 15 with OA (6 with AA features). All had residual tumor on postoperative CT scans. For nonanaplastic lesions, no local failure was seen at a median of 125 months (range, 28-240 months) in 6 patients who received postoperative radiotherapy to $60 \mathrm{~Gy}$ and chemotherapy with PCV. Nine patients who had no postoperative chemotherapy experienced local failure (median time to failure, 25 months). The 6 patients treated with 50 Gy had recurrence at a median of 36 months and $5(62 \%)$ of 8 patients treated to 60 Gy remained disease-free at a median of 66 months (3 had recurrence at 26, 40, and 60 months). Of the 9 patients with anaplastic tumor elements who were treated with 60 Gy and chemotherapy, 5 (56\%) had not had a recurrence at a median of 48 months.

Over the past several years, some major research has focused on the question of whether it is better to give a combination of PCV before radiotherapy or after radiotherapy at recurrence for AOs. Answering this question
V.A. Levin: Ten years of chemotherapy for gliomas

will be important, but of equal, if not greater, importance will be discovering and developing new chemotherapy approaches to the treatment of all oligodendroglial tumors. Because oligodendroglial tumors are uncommon, few studies of chemotherapy treatment for these tumors have been conducted over the past decade, and they have included small numbers of patients. Nonetheless, these trials can be instructive and will be reviewed.

The London Ontario Group did a retrospective analysis of 23 patients and found that a combination of etoposide and cisplatin had some palliative activity in patients who had tumor progression while on PCV (Peterson et al., 1996).

Fulton et al. (1996) evaluated oral etoposide $50 \mathrm{mg}$. $\mathrm{m}^{-2} \cdot$ day $^{-1}$ given until the neutrophil count dropped to $<1000 / \mu \mathrm{l}$ or the platelet count fell to $<75,000 / \mu \mathrm{l}$ and resumed once the counts had risen to normal levels. Fulton et al. (1996) treated 9 patients with AO who had a median TTP of 17 weeks, which is nearly twice that observed for patients with AA.

A study of paclitaxel treatment of 20 patients with recurrent oligodendroglioma found response in 3 patients and stable disease in 7 patients; the median duration of response and stable disease was 10 months (range, 5-14) (Chamberlain and Kormanik, 1997).

Trials of adjuvant chemotherapy (mostly with PCV) in adults have shown that more patients with $\mathrm{OA}$ than patients with AO have 2-year progression-free survival $(80$ vs. $56 \%$ ). When the survival and response to therapy of patients with OA of varying grades of malignancy were reviewed, Levin et al. (1997) found that patients experienced recurrence at a median of 1.8 years after initial presentation, and with additional chemotherapy and surgery $(20 \%)$, they survived a median of an additional 1.6 years. Of note was the fact that $25 \%$ of patients survived 6.9 years.

Kyritsis et al. (1997) found that of 12 patients with AO who were initially treated with nitrosourea-based therapy and 6 patients who were treated with various therapies at recurrence, 5 had a response and 7 had stable disease with most responses lasting longer than 12 months. Among patients with recurrent disease, 1 had a response and 5 had stable disease; the median TTP was 6 months.

Limited information is available on chemotherapy in children with oligodendrogliomas. The Children's Cancer Group protocol 945 included 17 patients aged from 2 to 21 years who were considered to have OA (Hariharan et al., 1996). All were treated with nitrosoureabased chemotherapy. Their 5 -year progression-free survival rate was $64 \%$. In comparison to $\mathrm{AO}$, the number of patients without tumor progression at 2 years was higher for oligodendroglioma patients than for $\mathrm{AO}$ patients ( 80 vs. $56 \%$ ). Hariharan et al. (1996) suggest treating children and adults with a PCV combination until a better therapy proves effective.

Summary. At present, the appropriate use of chemotherapy in the treatment of oligodendroglioma is primarily for recurrence. Although chemotherapy treatment for residual and recurrent $\mathrm{AO}$ and OA seems logi$\mathrm{cal}$, data to date present a mixed picture depending on 
factors such as patient age, diagnostic criteria, extent of surgery, and type of chemotherapy administered. At present, therapy based on PCV appears to be the most effective therapy among those tried. As more effective and less toxic forms of chemotherapy become available, most patients with $\mathrm{AO}$ or OA would in fact initially be treated with chemotherapy for long-term tumor control.

\section{The Special Case of Chemotherapy for Low-grade Gliomas}

Chemotherapy for low-grade gliomas has received little attention until this decade. Small studies have been conducted during which chemotherapy was given to avoid or defer irradiation of the CNS in children. Most of the studies have used carboplatin and vincristine (Packer et al., 1997) or nitrosourea combinations (Berger et al., 1995; Prados et al., 1997) with good results. Packer et al. (1997) reported on 78 children with a mean age of 3 years and with low-grade gliomas (32 fibrillary, 17 pilocytic, 3 mixed, and 26 histologic types undetermined) and tumors located primarily in the diencephalon $(n=58)$ and brain stem $(n=12)$. The children were treated at recurrence or after initial surgery or both with a 10week course of 2 cycles of carboplatin weekly for 4 weeks with concurrent weekly vincristine. The investigators found a progression-free survival rate of $68 \%$ at 3 years and a projected 5-year overall survival rate of $\sim 40 \%$ (Packer et al., 1997). Prados et al. (1997) treated 42 children (mean age 5 years) with a combination of 6thioguanine, procarbazine, mitolactol (dibromodulcitol), lomustine, and vincristine, and found a median 5year survival rate of $78 \%$.

Many patients with low-grade gliomas are children with chiasmal-hypothalamic astrocytomas. Petronio et al. (1991) reported 19 children (aged from 15 weeks to 15.6 years) with chiasmal/hypothalamic gliomas treated with chemotherapy after either surgical or radiological diagnosis. Twelve patients were treated immediately after diagnosis because of progressive symptoms and 7 received chemotherapy after either radiographic evidence of progression or clinical deterioration, which included progressive visual loss or intracranial hypertension. Twelve of the 19 tumors were biopsied ( 7 were classified as juvenile pilocytic astrocytomas, 2 as astrocytomas, 2 as $\mathrm{AA}$, and 1 as subependymal giant-cell astrocytoma). Eighty-three percent of the children were treated with a combination of 6-thioguanine, procarbazine, mitolactol, lomustine, and vincristine. Fifteen $(83 \%)$ of the 18 evaluable patients initially treated with chemotherapy either responded or experienced stabilization of their condition. Median TTP had not been reached at a median follow-up of 79 weeks (range, 6.6-303 weeks) and no tumor-related death had occurred.

Gajjar et al. (1993) reported on 13 children with infiltrative low-grade gliomas treated with alkylating agent or platinum-based chemotherapy regimens. In 11 patients, this was the initial postoperative treatment modality and, in 2 others, treatment for progressive disease after radiotherapy. Despite good initial responses, 5 of 6 patients who received no further treatment following chemotherapy (4 had a response and 1 had stable disease) developed progressive disease 5-13 months after completing chemotherapy. The remaining four patients with objective responses or stable disease responded to further treatment with radiotherapy or surgery.

Pons et al. (1992) reported on 14 of 20 patients (aged from 9 months to 20 years) with low-grade astrocytoma who completed one 8-week course of vincristine and etoposide. In a difficult-to-follow study, 4 patients exhibited partial or minor responses that were maintained for 10-35 months and 10 had stable disease for 10-42 months.

Among patients treated according to an NCOG protocol of BrdU during conventional radiotherapy followed by PCV for 1 year or until recurrence, 22 had low-grade contrast-enhancing (by CT) infiltrative astrocytoma. These patients had a 4-year progression-free survival rate of $68 \%$ and a 6 -year overall survival rate of $79 \%$ (Levin et al., 1995b).

Summary. It is clear that infiltrative low-grade astrocytomas and oligodendrogliomas are sensitive to various chemotherapies. Historically, nitrosourea-based therapies have dominated in adult and childhood clinical trials, though platinum and vincristine therapies have been found useful in childhood low-grade gliomas. Because these tumors respond to various chemotherapy approaches, when more effective and less toxic forms of chemotherapy become available, most patients with lowgrade astrocytoma and oligodendroglioma initially will be treated for long-term tumor control. With luck, we may even eliminate treatment with radiotherapy in the future.

\section{Current and New Cytotoxic Drugs for Gliomas}

Only two drug chemotypes are approved in the United States and Canada for the treatment of gliomas: carmustine and lomustine. In addition, practice patterns have led to the following drugs being approved for third party reimbursement: procarbazine, cisplatin, carboplatin, etoposide, and nitrogen mustard. In this section, I will discuss some of these drugs, some newer drugs, and some of the drug combinations evaluated in phase II studies.

Temozolomide (Temodal) is an oral agent that readily crosses the blood-brain barrier and biotransforms to monomethyl triazenoimidazole carboxamide, an alkylating species that methylates the $\mathrm{O} 6$ position of guanine. In the near future, Temodal is expected to gain Food and Drug Administration approval for the treatment of recurrent GBM and AA. A phase II study of 161 patients with AG at first relapse showed a $42 \%$ response rate with $24 \%$ of patients experiencing stable disease; on an intent-to-treat basis, the TTP was $46 \%$ $(95 \%$ CI $37 \%, 56 \%)$ at 6 months and $25 \%$ at 12 months (Levin et al., 1997).

Taxol (Paclitaxel), a drug highly active against adenocarcinomas, shows modest activity against recurrent primary CNS tumors with acceptable toxicity (Chamberlain and Kormanik, 1995, 1997; Chang et al., 1996). Complicating the evaluation of this agent in patients with CNS tumors has been its accelerated plasma clearance in patients receiving anticonvulsants (Chang et al., 1996).

Another drug, tamoxifen, is active in the treatment of breast adenocarcinoma. It is a nonspecific protein 
kinase $\mathrm{C}$ inhibitor and has been evaluated for the treatment of recurrent high-grade glial neoplasms. The rationale is that tumor cells, including glioma cells, have high levels of protein kinase $\mathrm{C}$ activity compared with normal astrocytes. While the use of tamoxifen as a chemotherapeutic agent for high-grade gliomas is controversial, reports suggest that a subgroup of patients have a median survival from initiation of tamoxifen treatment of 69 weeks for AA and 31 weeks for GBM (Couldwell et al., 1996). Studies are also under way to evaluate tamoxifen in combination with other anticancer agents. One study evaluated the use of carboplatin and tamoxifen after surgery and radiotherapy in 40 patients, but could not demonstrate increased efficacy compared with a historical series (Mastronardi et al., 1998).

Etoposide (VP-16) is a topoisomerase II inhibitor that is restricted in its ability to cross the intact bloodbrain barrier (Levin, 1980; Levin et al., 1976). Because of the slow doubling of gliomas, etoposide has been administered most recently as an oral treatment over 21 days or longer. In a study of chronic low-dose treatment of $50 \mathrm{mg} / \mathrm{m}^{2}$, Fulton et al. (1996) found little activity against malignant astrocytomas in adults, though more activity was seen in patients with $\mathrm{AO}$. On the other hand, in 14 children with supratentorial malignant gliomas, Chamberlain (1997) found that chronic oral etoposide for 21 days produced partial responses in 3 patients and stable disease in 4; the median TTP was 8 months.

Etoposide was combined with carboplatin in a study of 31 patients with high-grade gliomas (Ameri et al., 1997). The investigators reported a response rate of $13 \%$, a stable disease rate of $32 \%$, and a median TTP for this subgroup of 28 weeks. In a comparable study, Jeremic et al. (1992) treated patients for 3 months before evaluating for response (when possible), and reported response rates of $21 \%$, stable disease rates of $32 \%$, and a median TTP of nearly 48 weeks.

The use of etoposide with cisplatin was evaluated in 36 patients with recurrent high-grade astrocytomas (Buckner et al., 1990). Thirty-one percent of patients responded $(n=6)$ or had stable disease $(n=5)$; median TTP for this subgroup was 6 months.

Mitolactol an older alkylating agent, has been evaluated in various chemotherapy regimens over the past decade. It was combined with lomustine and procarbazine in a study of 26 patients with recurrent malignant gliomas. The response rate was $55 \%$ in AA compared with $12 \%$ in GBM (Hildebrand et al., 1998). In another study, mitolactol was combined with oral 6thioguanine, procarbazine, lomustine, 5-fluorouracil, and hydroxyurea for treatment of recurrent GBM (37 patients) or AG (38 patients) (Levin and Prados, 1992). There was a nonstatistically significant difference on the basis of whether patients had recurrence after radiotherapy alone or radiotherapy and a nitrosourea. For patients with GBM who had received radiotherapy alone, the rate of response or stable disease was $61 \%$ and the median TTP was 9.3 months. For patients with GBM who had received radiotherapy and a nitrosourea, the rate of response or stable disease was
$58 \%$ and the median TTP was 5.1 months. Corresponding values for patients with AG who had received radiotherapy alone or radiotherapy and a nitrosourea were $92 \%$ and 15 months and $38 \%$ and 10.6 months.

Another old drug recently restudied is procarbazine. Thirty-five patients in whom treatment with radiation and a nitrosourea failed were treated with oral procarbazine for 4-week periods every 8 weeks (Newton et al., 1990). The partial response rate of patients was $20 \%$ and the stable disease rate was $31 \%$. These data are similar to those reported earlier by Rodriguez et al. (1989).

Single-agent high-dose carboplatin has also been evaluated against recurrent malignant gliomas. Yung et al. (1991a) treated 29 evaluable patients with 400-450 $\mathrm{mg} / \mathrm{m}^{2}$ every 4 weeks; $48 \%$ of patients had response or stable disease and had a median TTP of 26 weeks. In another dose-finding study, 32 patients were treated with carboplatin every 3 weeks at a dose of $350 \mathrm{mg} / \mathrm{m}^{2}$, with a dose escalation every 6 weeks until level 4 hematologic toxicity was reached. Of the 28 patients who could be evaluated for a response, $50 \%$ demonstrated a response or had stabilization of their disease after 2 infusions of carboplatin; the median TTP was 19 weeks (Warnick et al., 1994).

Single-agent difluormethylornithine (Eflornithine), an irreversible ornithine decarboxylase inhibitor, was evaluated in 36 patients with GBM and 44 patients with AG (Levin et al., 1992). Partial or minor response or stable disease was seen in $45 \%$ of patients with AG, and the median TTP was 49 weeks; however, only $17 \%$ of patients with GBM had response or stable disease, and the median TTP had not been reached by a median follow-up of 32 weeks. The study suggested that eflornithine is an effective palliative therapy for recurrent AG.

Oral tauromustine, $130 \mathrm{mg} / \mathrm{m}^{2}$ every 5 weeks, was evaluated in a phase II study in 46 patients (Gregor et al., 1992). All patients were followed until death and none received second-line chemotherapy. The median posttreatment survival was 26 weeks for patients with GBM and 57 weeks for patients with AA. The overall 2 -year survival rate was $23 \%$ for patients with GBM and $69 \%$ for patients with AA.

Another new drug, crisnatol mesylate was evaluated in 6 patients with recurrent $\mathrm{AA}$ and 20 patients with GBM (New et al., 1997). The drug was administered as a 72-hour infusion every 21 days after radiotherapy and, in some cases, after chemotherapy. Neurologic side-effects were acute during drug infusion and were dose-limiting.

Topotecan is a topoisomerase I inhibitor that has been evaluated recently. A preliminary report of 26 patients treated at recurrence indicated that $12 \%$ responded and $15 \%$ had their disease stabilized with infusions of 21 days every 4 weeks (Kyritsis et al., 1997).

Summary. Few new cytotoxic drugs have become available for the treatment of gliomas during the past decade. The anticipated approval of Temodal this year will be the first Food and Drug Administration approval of a new drug chemotype for recurrent malignant gliomas since the early 1970s. Most energy has been focused in creative scheduling and combinations 
with other drugs. The results have been variable and sometimes encouraging. All therapies, nonetheless, remain palliative and few are likely to lead to randomized phase III trials.

\section{Biological Response Modifiers and Signaling-Specific Therapies}

Alpha and beta interferons are proteins with limited access to the brain because of the restriction of the blood-brain barrier. Nonetheless, the interferons have the capacity to slow the growth of CNS gliomas. The precise mechanism of these protein drugs is under study, but it is likely that their major action is to impair tumor angiogenesis or invasion or both. In a large sixcenter phase I/II trial, human recombinant interferon beta (Betaseron) was evaluated in patients with recurrent malignant glioma (Yung et al., 1991b). Betaseron was given intravenously three times per week, starting at $90 \mathrm{mIU}$ per dose and escalating by $90 \mathrm{mIU}$ every 2 weeks, to a maximum dose of $540 \mathrm{mIU}$ per treatment. Neurologic side effects were dose-limiting. Of the 41 patients with GBM and 24 with AA, 15 (23\%) had an objective response and $18(28 \%)$ had stable disease. The median TTP was 24 weeks for the responders, 10 weeks for the nonresponders, and 23 weeks for the whole group. In another study, 32 children with brain stem glioma were treated with recombinant beta interferon and 72 Gy of hyperfractionated radiation. The median TTP from study entry was 5 months and the median time to death was 9 months; these results are comparable to results obtained with irradiation only.

Interferon alpha has been used in combination with nitrosoureas (Brandes et al., 1997; Buckner et al., 1995), eflornithine (Buckner et al., 1998), and oral tamoxifen (Chang et al., 1998) without clear indication of either additive or synergistic benefit. Interferon alpha with tamoxifen was poorly tolerated because of neurologic side effects and led to early closure of the study (Chang et al., 1998).

Intratumoral recombinant interferon gamma has also been evaluated in a setting adjuvant to surgery and external irradiation in a small study of 21 patients with GBM patients randomly assigned to receive intratumoral interferon (Farkkila et al., 1994). There were no differences in the survival times (55 vs. 54 weeks).

The retinoids have also been investigated in patients with malignant glioma because these agents inhibit cellular proliferation and decrease epidermal growth factor receptor-mediated tyrosine kinase activity in several glioma cell lines. Thirty-six patients with recurrent malignant glioma were treated with tretinoin (all-transretinoic acid) at $120-150 \mathrm{mg} \cdot \mathrm{m}^{-2} \cdot \mathrm{day}^{-1}$ for 3 weeks followed by a 1 -week rest (Kaba et al., 1997). One (3\%) of 34 evaluable patients had a minor response and 14 $(41 \%)$ had stable disease. The median TTP for the patients with stable disease and the single responder was 17 weeks. Though the $150 \mathrm{mg} \cdot \mathrm{m}^{-2} \cdot$ day $^{-1}$ was associated with a high incidence of headache, the $120 \mathrm{mg}$. $\mathrm{m}^{-2} \cdot$ day $^{-1}$ dose was well tolerated with mild, mainly dermatologic side effects. Another retinoid, isotretinoin (13-cis-retinoic acid), was evaluated at a dosage of 50 $\mathrm{mg} / \mathrm{m}^{2}$ twice daily for 3 weeks with a 1 -week rest between cycles (Yung et al., 1996). Of the 43 patients with recurrent glioma treated (15 with GBM, 15 with $\mathrm{AA}$, and 13 with other malignant gliomas), 10 (23\%) responded and $13(30 \%)$ had stable disease for a median 48 weeks. The median TTP for all patients with GBM was 19 weeks and for those with AG, 11 weeks.

A preliminary report of an aborted randomized phase II study of recombinant interferon beta $(6 \mathrm{mIU}$ subcutaneously 3 times a week) and cis-retinoic acid $\left(50 \mathrm{mg} / \mathrm{m}^{2}\right.$ twice daily for 3 weeks every 4 weeks) versus cis-retinoic acid alone is disappointing (Yung et al., 1994). After the first 65 patients, the interferon dose was increased to 18 mIU. There were 94 evaluable patients. The rate of patients with partial response or stable disease was $61 \%$ for $\mathrm{AA}$ and $57 \%$ for GBM. Response was less common than stable disease. Twenty-five percent (11 of 44) of patients with AA remained on treatment for 15-49 weeks and $18 \%$ (9 of 50) of patients with GBM remained on treatment for 12-64 weeks. No synergism between 13-cis-retinoic acid and interferon beta was shown, and increasing the interferon beta dose $18 \mathrm{mIU}$ did not affect response rate or duration.

There is great anticipation that drugs that inhibit tumor angiogenesis will help control the growth and invasion of gliomas and other cancers. Unfortunately, pure anti-angiogenesis drugs are unavailable for human trials. Fine et al. (1997) reported their preliminary findings from a phase II trial of oral thalidomide in patients with recurrent high-grade gliomas. Thalidomide use $(1200 \mathrm{mg} /$ day) was evaluated in patients with recurrent high-grade astrocytomas and mixed gliomas. Of the 32 patients reported, all were evaluable for side effects and 10 were evaluable for response. Major drug side effects included somnolence (three patients) and drug rash (two patients). Minimal radiographic responses were seen in $20 \%$ ( 2 of 10), and one patient remained on thalidomide for more than 7 months.

Much pharmaceutical industry focus during the past decade has been on the development of small molecule inhibitors of specific signaling pathways. A product of that effort is SU101, a potent inhibitor of platelet-derived growth factor-mediated cell signaling. A recently completed phase I toxicity and pharmacokinetic study has defined the dose schedule for phase II and III studies (Malkin et al., 1996). Given the 1-hour plasma half-life for SU101 and the 400-hour plasma half-life for the major metabolite SU20, a 24-hour continuous intravenous infusion weekly for 4 weeks appears appropriate. Of the 12 patients treated in the phase I trial, 3 patients had a minor response.

\section{The Bright Future}

Future therapy for our patients with cancer of the CNS is certain to improve markedly over the next decade. Through understanding the cancer genome and critical signaling pathways, we will better understand ways to stop cancer growth. Unfortunately, barring some unforeseen breakthrough, that knowledge will precede by many years 
our capacity actually to create the necessary drugs. Eventually, however, we will treat our patients with selective and specific drugs for each type of CNS malignancy. I believe that our future therapies will block unique tumorspecific signaling pathways. An enormous amount of pharmaceutical research dollars is being invested to find potential drugs for the treatment of cancer that inhibit specific signaling pathways. It is likely that more than one sig- nal-inhibiting drug will be used with a conventional cytotoxic drug to increase tumor cell stress and force cells into an apoptotic death spiral or permanent growth arrest.

\section{Acknowledgment}

I thank Ms. Stephanie Deming for her editorial assistance.

\section{References}

Allison, R.R., Schulsinger, A., Vongtama, V., Barry, T., and Shin, K.H. (1997) Radiation and chemotherapy improve outcome in oligodendroglioma. Int. J. Radiat. Oncol. Biol. Phys. 37, 399-403.

Ameri, A., Poisson, M., Chauveinc, L., Chen, Q.M., and Delattre, J.Y. (1997) Treatment of recurrent malignant supratentorial gliomas with the association of carboplatin and etoposide: A phase II study. J. Neurooncol. 32 , 155-160.

Berger, M.S., Leibel, S.A., Bruner, J.M., Finlay, J.L., and Levin, V.A. (1995) Primary central nervous system tumors of the supratentorial compartment. In: Levin, V.A. (Ed.), Cancer in the Nervous System. New York:Churchill Livingstone. pp. 57-125.

Boiardi, A., Silvani, A., Milanesi, I., Botturi, M., and Broggi, G. (1992) Carboplatin combined with carmustine and etoposide in the treatment of glioblastoma. Ital. J. Neurol. Sci. 13, 717-722.

Brandes, A.A., Scelzi, E., Zampieri, P., Rigon, A., Rotilio, A., Amista, P., Berti, F., and Fiorentino, M.V. (1997) Phase II trial with BCNU plus alpha-interferon in patients with recurrent high-grade gliomas. Am. J. Clin. Oncol. 20, 364-367.

Brandes, A.A., Rigon, A., Zampieri, P., Ermani, M., Carollo, C., Altavilla, G., Turazzi, S., Chierichetti, F., and Fiorentino, M.V. (1998) Carboplatin and teniposide concurrent with radiotherapy in patients with glioblastoma multiforme - A phase II study. Cancer 82, 355-361.

Buckner, J.C., Brown, L.D., Cascino, T.L., Gerstner, J.B., Krook, J.E., Westberg, M.W., Wiesenfeld, M., O'Fallon, J.R., and Scheithauer, B. (1990) Phase II evaluation of infusional etoposide and cisplatin in patients with recurrent astrocytoma. J. Neurooncol. 9, 249-254.

Buckner, J.C., Brown, L.D., Kugler, J.W., Cascino, T.L., Krook, J.E., Mailliard, J.A., Kardinal, C.G., Tschetter, L.K., O'Fallon, J.R., and Scheithauer, B.W. (1995) Phase II evaluation of recombinant interferon alpha and BCNU in recurrent glioma. J. Neurosurg. 82, 430-435.

Buckner, J.C., Burch, P.A., Cascino, T.L., Ofallon, J.R., and Scheithauer, B.W. (1998) Phase II trial of recombinant interferon-alpha-2a and eflornithine in patients with recurrent glioma. J. Neurooncol. 36, 65-70.

Cairncross, G., Macdonald, D., Ludwin, S., Lee, D., Cascino, T., Buckner, J., Fulton, D., Dropcho, E., Stewart, D., and Schold, C.J. (1994) Chemotherapy for anaplastic oligodendroglioma. National Cancer Institute of Canada Clinical Trials Group. J. Clin. Oncol. 12, 2013-2021.

Chamberlain, M.C. (1997) Recurrent supratentorial malignant gliomas in children. Long-term salvage therapy with oral etoposide. Arch. Neurol. 54, 554-558.

Chamberlain, M.C., and Kormanik, P. (1995) Salvage chemotherapy with paclitaxel for recurrent primary brain tumors. J. Clin. Oncol. 13, 2066-2071.

Chamberlain, M.C., and Kormanik, P.A. (1997) Salvage chemotherapy with paclitaxel for recurrent oligodendrogliomas. J. Clin. Oncol. 15, 3427-3432.

Chang, S., Schold, C., Spence, A., Berger, M., McAllister, L., Mehta, M., Gilbert, M.R., Fulton, D., and Prados, M. (1996) Phase I/II study of paclitaxel chemotherapy for recurrent malignant glioma: Preliminary report.
North American Brain Tumor Consortium. Proc. Annu. Meet. Am. Soc. Clin. Oncol. 15, A276. (Abstract)

Chang, S.M., Barker, F.G., Huhn, S.L., Nicholas, M.K., Page, M., Rabbitt, J., and Prados, M.D. (1998) High dose oral tamoxifen and subcutaneous interferon alpha-2a for recurrent glioma. J. Neurooncol. 37, 169-176.

Chauveinc, L., Sola-Martinez, M.T., Martin-Duverneuil, M., Mazeron, J.J., Faillot, T., Cornu, P., Capelle, L., Delattre, J.Y., Poisson, M., Baillet, F., and Chiras, J. (1996) Intra-arterial chemotherapy with ACNU and radiotherapy in inoperable malignant gliomas. J. Neurooncol. 27, 141-147.

Choucair, A.K., Levin, V.A., Gutin, P.H., Davis, R.L., Silver, P., Edwards, M.S., and Wilson, C.B. (1986) Development of multiple lesions during radiation therapy and chemotherapy in patients with gliomas. J. Neurosurg. 65 , 654-658.

Corn, B.W., Yousem, D.M., Scott, C.B., Rotman, M., Asbell, S.O., Nelson, D.F., Martin, L., and Curran, W.J., Jr. (1994) White matter changes are correlated significantly with radiation dose. Observations from a randomized dose-escalation trial for malignant glioma. (Radiation Therapy Oncology Group 83-02) Cancer 74, 2828-2835.

Couldwell, W.T., Hinton, D.R., Surnock, A.A., DeGiorgio, C.M., Weiner, L.P., Apuzzo, M.L., Masri, N.L., Law, R.E., and Weiss, M.H. (1996) Treatment of recurrent malignant gliomas with chronic oral high-dose tamoxifen. Clin. Cancer Res. 2, 619-622.

Dolan, M.E., Mitchell, R.B., Mummert, C., Moschel, R.C., and Pegg, A.E. (1991) Effect of O6-benzylguanine analogues on sensitivity of human tumor cells to the cytotoxic effects of alkylating agents. Cancer Res. 51, 3367-3372.

Dolan, M.E., Pegg, A.E., Moschel, R.C., Vishnuvajjala, B.R., Flora, K.P., Grever, M.R., and Friedman, H.S. (1994) Biodistribution of O6-benzylguanine and its effectiveness against human brain tumor xenografts when given in polyethylene glycol or cremophor-EL. Cancer Chemother. Pharmacol. 35 , 121-126.

Dropcho, E.J., Rosenfeld, S.S., Vitek, J., Guthrie, B.L., and Morawetz, R.B. (1998) Phase II study of intracarotid or selective intracerebral infusion of cisplatin for treatment of recurrent anaplastic gliomas. J. Neurooncol. 36, 191-198.

Dunkel, I.J., O'Malley, B., and Finlay, J.L. (1996) Is there a role for high-dose chemotherapy with stem cell rescue for brain stem tumors of childhood? Pediatr. Neurosurg. 24, 263-266.

Farkkila, M., Jaaskelainen, J., Kallio, M., Blomstedt, G., Raininko, R., Virkkunen, P., Paetau, A., Sarelin, H., and Mantyla, M. (1994) Randomised, controlled study of intratumoral recombinant gamma-interferon treatment in newly diagnosed glioblastoma. Brit. J. Cancer 70, 138-141.

Fernandez-Hidalgo, O.A., Vanaclocha, V., Vieitez, J.M., Aristu, J.J., Rebollo, J., Gurpide, A., Aramendia, J.M., Moreno-Palanques, R., Martin-Algarra, S., Subira, M.L., and Brugarolas, A. (1996) High-dose BCNU and autologous progenitor cell transplantation given with intra-arterial cisplatinum and simultaneous radiotherapy in the treatment of high-grade gliomas: Benefit for selected patients. Bone Marrow Transplant. 18, 143-149.

Fine, H.A., Loeffler, J.S., Kyritsis, A., Wen, P., Black, P.M., Levin, V.A., Graham, S., and Yung, W.A. (1997) A phase II trial of the anti-angiogenic agent, 
thalidomide, in patients with recurrent high-grade gliomas. Proc. Annu. Meet. Am. Soc. Clin. Oncol. 16, A1372. (Abstract)

Finlay, J.L. (1996) The role of high-dose chemotherapy and stem cell rescue in the treatment of malignant brain tumors. Bone Marrow Transplant. 18 (suppl 3), S1-S5.

Finlay, J.L., August, C., Packer, R., Zimmerman, R., Sutton, L., Freid, A., Rorke, L., Bayever, E., Kamani, N., and Kramer, E. (1990) High-dose multi-agent chemotherapy followed by bone marrow 'rescue' for malignant astrocytomas of childhood and adolescence. J. Neurooncol. 9, 239-248.

Finlay, J.L., Boyett, J.M., Yates, A.J., Wisoff, J.H., Milstein, J.M., Geyer, J.R., Bertolone, S.J., McGuire, P., Cherlow, J.M., and Tefft, M. (1995) Randomized phase III trial in childhood high-grade astrocytoma comparing vincristine, lomustine, and prednisone with the eight-drugs-in-1-day regimen. Childrens Cancer Group. J. Clin. Oncol. 13, 112-123.

Finlay, J.L., Goldman, S., Wong, M.C., Cairo, M., Garvin, J., August, C., Cohen, B.H., Stanley, P., Zimmerman, R.A., Bostrom, B., Geyer, J.R., Harris, R.E., Sanders, J., Yates, A.J., Boyett, J.M., and Packer, R.J. (1996) Pilot study of high-dose thiotepa and etoposide with autologous bone marrow rescue in children and young adults with recurrent CNS tumors. The Children's Cancer Group. J. Clin. Oncol. 14, 2495-2503.

Fulton, D., Urtasun, R., and Forsyth, P. (1996) Phase II study of prolonged oral therapy with etoposide (VP16) for patients with recurrent malignant glioma. J. Neurooncol. 27, 149-155.

Gajjar, A., Heideman, R.L., Kovnar, E.H., Langston, J.A., Sanford, R.A., Douglass, E.C., Jenkins, J.J., Horowitz, M.E., and Kun, L.E. (1993) Response of pediatric low grade gliomas to chemotherapy. Pediatr. Neurosurg. 19, 113-118.

Gregor, A., Rampling, R., Aapro, M., Malmstrom, P., Whittle, I.R., Rye, R., Stewart, D., Sellar, R., Demierre, B., and Ironside, J.W. (1992) Phase II study of tauromustine in malignant glioma. Eur. J. Cancer 28A, 1959-1962.

Grossman, S.A., Wharam, M., Sheidler, V., Kleinberg, L., Zeltzman, M., Yue, N., and Piantadosi, S. (1997) Phase II study of continuous infusion carmustine and cisplatin followed by cranial irradiation in adults with newly diagnosed high-grade astrocytoma. J. Clin. Oncol. 15, 2596-2603.

Hammack, J., Shaw, E., Ivnik, R., Arusell, R., Novotny, P., and O'Fallon, J. (1995) Neurocognitive function in patients receiving radiation therapy (RT) for supratentorial low grade glioma (LGG): A North Central Cancer Treatment Group (NCCTG) prospective study. Proc. Annu. Meet. Am. Soc. Clin. Oncol. 14, A299. (Abstract)

Hariharan, S., Stuebgen, P., Boyett, J.M., Yates, A.J., and Finlay, J.L. (1996) Anaplastic mixed gliomas in childhood: Results from the Children's Cancer Group Study, CCG-945. Proc. Annu. Meet. Am. Soc. Clin. Oncol. 15, A1471. (Abstract)

Heideman, R.L., Kuttesch, J.J., Gajjar, A.J., Walter, A.W., Jenkins, J.J., Li, Y., Sanford, R.A., and Kun, L.E. (1997) Supratentorial malignant gliomas in childhood: A single institution perspective. Cancer 80, 497-504.

Hellman, R., Neuberg, D.S., Wagner, H., Gunnet, M., Robins, H.I., Karp, D., Flynn, P., and Adams, G. (1998) A therapeutic trial of radiation therapy with vincristine, etoposide, and procarbazine (VVP) in high grade intracranial gliomas-An Eastern Cooperative Oncology Group study (E2392). J. Neurooncol. 37, 55-62.

Hiesiger, E.M., Green, S.B., Shapiro, W.R., Burger, P.C., Selker, R.G., Mahaley, M.S., Jr., Ransohoff, J., VanGilder, J.C., Mealey, J.J., and Robertson, J.T. (1995) Results of a randomized trial comparing intra-arterial cisplatin and intravenous PCNU for the treatment of primary brain tumors in adults: Brain Tumor Cooperative Group trial 8420A. J. Neurooncol. 25, 143-154.

Hildebrand, J., Dewitte, O., and Sahmoud, T. (1998) Response of recurrent glioblastoma and anaplastic astrocytoma to dibromodulcitol, BCNU and procarbazine - A phase-II study. J. Neurooncol. 37, 155-160.

Ikeda, J., Aida, T., Sawamura, Y., Abe, H., Kaneko, S., Kashiwaba, T., Kawamoto, T., Mitsumori, K., and Saitoh, H. (1996) Phase II study of
DTIC, ACNU, and vincristine combination chemotherapy for supratentorial malignant astrocytomas. Neurol. Med. Chir. 36, 555-558.

Iwadate, Y., Namba, H., and Sueyoshi, K. (1995) Intra-arterial ACNU and cisplatin chemotherapy for the treatment of glioblastoma multiforme. Neurol. Med. Chir. 35, 598-603.

Jason, G.W., Pajurkova, E.M., Taenzer, P.A., and Bultz, B.D. (1997) Acute effects on neuropsychological function and quality of life by high-dose multiple daily fractionated radiotherapy for malignant astrocytomas: Assessing the tolerability of a new radiotherapy regimen. Psycho-Oncology 6, 151-157.

Jeremic, B., Grujicic, D., Jevremovic, S., Stanisavljevic, B., Milojevic, L., Djuric, L., and Mijatovic, L. (1992) Carboplatin and etoposide chemotherapy regimen for recurrent malignant glioma: A phase II study. J. Clin. Oncol. 10, 1074-1077.

Kaba, S.E., Kyritsis, A.P., Conrad, C., Gleason, M.J., Newman, R., Levin, V.A., and Yung, W.K. (1997) The treatment of recurrent cerebral gliomas with all-trans-retinoic acid (tretinoin). J. Neurooncol. 34, 145-151.

Kirby, S., Macdonald, D., Fisher, B., Gaspar, L., and Cairncross, G. (1996) Pre-radiation chemotherapy for malignant glioma in adults. Can. J. Neurol. Sci. 23, 123-127.

Knerich, R., Adinolfi, D., Giunta, F., Buoncristiani, P., Gaetani, P., Assietti, R., D'Ettorre, F., Butti, G., and Schiffer, D. (1990) Single versus multiple drug therapy in the combined treatment of malignant gliomas. A multicenter study. J. Neurosurg. Sci. 34, 251-255.

Kurpad, S.N., Dolan, M.E., McLendon, R.E., Archer, G.E., Moschel, R.C., Pegg, A.E., Bigner, D.D., and Friedman, H.S. (1997) Intraarterial O6-benzylguanine enables the specific therapy of nitrosourea-resistant intracranial human glioma xenografts in athymic rats with 1,3-bis(2-chloroethyl)-1nitrosourea. Cancer Chemother. Pharmacol. 39, 307-316.

Kyritsis, A.P., Yung, W.K., Bruner, J., Gleason, M.J., and Levin, V.A. (1993) The treatment of anaplastic oligodendrogliomas and mixed gliomas. Neurosurgery 32, 365-370.

Kyritsis, A.P., Yung, W.K., Jaeckle, K.A., Bruner, J., Gleason, M.J., Ictech, S.E., Flowers, A., and Levin, V.A. (1996) Combination of 6-thioguanine, procarbazine, lomustine, and hydroxyurea for patients with recurrent malignant gliomas. Neurosurgery 39, 921-926.

Kyritsis, A., Newlands, E.S., Brock, C.S.G., Jaeckle, K., Levin, V., Bower, M., Evans, H., Dane, G., DeWitte, M., and Yung, W. (1997) Phase II trial of topotecan (T) as a continuous intravenous infusion (CIV) in patients (pts) with high-grade gliomas. Proc. Annu. Meet. Am. Soc. Clin. Oncol. 16, A1404. (Abstract)

Levin, V.A. (1980) Relationship of octanol/water partition coefficient and molecular weight to rat brain capillary permeability. J. Med. Chem. 23, 682-684.

Levin, V.A., and Prados, M.D. (1992) Treatment of recurrent gliomas and metastatic brain tumors with a polydrug protocol designed to combat nitrosourea resistance. J. Clin. Oncol. 10, 766-771.

Levin, V.A., Landahl, H.D., and Freeman-Dove, M.A. (1976) The application of brain capillary permeability coefficient measurements to pathological conditions and the selection of agents which cross the blood-brain barrier. J. Pharmacokinet. Biopharm. 4, 499-519.

Levin, V.A., Silver, P., Hannigan, J., Wara, W.M., Gutin, P.H., Davis, R.L., and Wilson, C.B. (1990) Superiority of post-radiotherapy adjuvant chemotherapy with $\mathrm{CCNU}$, procarbazine, and vincristine (PCV) over BCNU for anaplastic gliomas: NCOG 6G61 final report. Int. J. Radiat. Oncol. Biol. Phys. 18, 321-324.

Levin, V.A., Prados, M.D., Yung, W.K., Gleason, M.J., Ictech, S., and Malec, M. (1992) Treatment of recurrent gliomas with eflornithine. J. Nat. Cancer Inst. 84, 1432-1437.

Levin, V.A., Maor, M.H., Thall, P.F., Yung, W.K., Bruner, J., Sawaya, R., Kyritsis, A.P., Leeds, N., Woo, S., and Rodriguez, L. (1995a) Phase II study of accelerated fractionation radiation therapy with carboplatin followed by 
vincristine chemotherapy for the treatment of glioblastoma multiforme. Int. J. Radiat. Onco. Biol. Phys. 33, 357-364.

Levin, V.A., Prados, M.D., Wara, W.M., Davis, R.L., Gutin, P.H., Phillips, T.L., Lamborn, K., and Wilson, C.B. (1995b) Radiation therapy and bromodeoxyuridine chemotherapy followed by procarbazine, lomustine, and vincristine for the treatment of anaplastic gliomas. Int. J. Radiat. Oncol. Biol. Phys. 32, 75-83.

Levin, V.A., Leibel, S.A., and Gutin, P.H. (1997) Neoplasms of the central nervous system. In: DeVita, V.T., Jr., Hellman, S., and Rosenberg, S.A. (Eds.), Cancer: Principles and Practice of Oncology. Philadelphia: LippincottRaven. pp. 2022-2082.

Malkin, M.G., Green, S.B., Byar, D.P., Strike, T.A., Burger, P.C., Vogel, F.S., Pistenmaa, D.A., Mahaley, M.S.J., Ransohoff, J., and Shapiro, W.R. (1994) Superiority of $\mathrm{PCNU}$ over $\mathrm{AZQ}$ in the treatment of primary brain tumors: Results of a prospective randomized trial (81-20) by the Brain Tumor Study Group. J. Neurooncol. 22, 55-65.

Malkin, M.G., Mason, W.P., Lieberman, F.S., Shawver, L.K., and Hannah, A.L. (1996) A phase I and pharmacokinetic study of SU101, a novel signal transduction inhibitor in patients with recurrent malignant glioma. Proc. Annu. Meet. Am. Soc. Clin. Oncol. 15, A1573. (Abstract)

Mason, W., Louis, D.N., and Cairncross, J.G. (1997) Chemosensitive gliomas in adults: Which ones and why? J. Clin. Oncol. 15, 3423-3426.

Mastronardi, L., Puzzilli, F., Couldwell, W.T., Farah, J.O., and Lunardi, P. (1998) Tamoxifen and carboplatin combination treatment of high-grade gliomas-Results of a clinical trial on newly diagnosed patients. J. Neurooncol. 38, 59-68.

Mineura, K., Izumi, I., Kuwahara, N., and Kowada, M. (1994) O6-methylguanine-DNA methyltransferase activity in cerebral gliomas. A guidance for nitrosourea treatment? Acta. Oncol. 33, 29-32.

New, P., Vokes, E., Rogers, L., Bazan, C., Hohneker, J., Eble, M., and Vilk, P. (1997) Long-term response to crisnatol mesylate in patients with glioma. Invest. New Drugs 15, 343-352.

Newton, H.B., Junck, L., Bromberg, J., Page, M.A., and Greenberg, H.S. (1990) Procarbazine chemotherapy in the treatment of recurrent malignant astrocytomas after radiation and nitrosourea failure. Neurology 40 , 1743-1746.

Nishimura, R., Takahashi, M., Morishita, S., Sumi, M., Uozumi, H., and Sakamoto, Y. (1992) MR imaging of late radiation brain injury. Radiat. Med. 10, 101-108.

Packer, R.J., Ater, J., Allen, J., Phillips, P., Geyer, R., Nicholson, H.S., Jakacki, Kurczynski, E., Needle, M., Finlay, J., Reaman, G., and Boyett, J.M. (1997) Carboplatin and vincristine chemotherapy for children with newly diagnosed progressive low-grade gliomas. J. Neurosurg. 86, 747-754.

Peterson, K., Paleologos, N., Forsyth, P., Macdonald, D.R., and Cairncross, J.G. (1996) Salvage chemotherapy for oligodendroglioma. J. Neurosurg. 85, 597-601.

Petronio, J., Edwards, M.S., Prados, M., Freyberger, S., Rabbitt, J., Silver, P., and Levin, V.A. (1991) Management of chiasmal and hypothalamic gliomas of infancy and childhood with chemotherapy. J. Neurosurg. 74, 701-708.

Phillips, T.L., Levin, V.A., Ahn, D.K., Gutin, P.H., Davis, R.L., Wilson, C.B., Prados, M.D., Wara, W.M., and Flam, M.S. (1991) Evaluation of bromodeoxyuridine in glioblastoma multiforme: A Northern California Cancer Center Phase II study. Int. J. Radiat. Oncol. Biol. Phys. 21, 709-714.

Pons, M.A., Finlay, J.L., Walker, R.W., Puccetti, D., Packer, R.J. and McElwain, M. (1992) Chemotherapy with vincristine (VCR) and etoposide (VP-16) in children with low-grade astrocytoma. J. Neurooncol. 14, 151-158.

Postma, T.J., Vangroeningen, C.J., Witjes, R.M., Weerts, J.E., Kralendonk, J.H., and Heimans, J.J. (1998) Neurotoxicity of combination chemotherapy with procarbazine, ccnu and vincristine (PCV) for recurrent glioma. J. Neurooncol. 38, 69-75.
Prados, M.D., Edwards, M.S., Rabbitt, J., Lamborn, K., Davis, R.L., and Levin, V.A. (1997) Treatment of pediatric low-grade gliomas with a nitrosoureabased multiagent chemotherapy regimen. J. Neurooncol. 32, 235-241.

Prados, M.D., Scott, C.B., Rotman, M., Rubin, P., Murray, K., Sause, W., Asbell, S., Comis, R., Curran, W., Nelson, J., Davis, R.L., Levin, V.A., Lamborn, K., and Phillips, T.L. (1998a) Influence of bromodeoxyuridine radiosensitization on malignant glioma patient survival: $\mathrm{A}$ retrospective comparison of survival data from the Northern California Oncology Group (NCOG) and Radiation Therapy Oncology Group (RTOG) trials for glioblastoma multiforme and anaplastic astrocytoma. Int. J. Radiat. Oncol. Biol. Phys. 40, 653-659.

Prados, M.D., Larson, D.A., Lamborn, K., Mcdermott, M.W., Sneed, P.K., Wara, W.M., Chang, S.M., Mack, E.E., Krouwer, H.G., Chandler, K.L., Warnick, R.E., Davis, R.L., Rabbitt, J.E., Malec, M., Levin, V.A., Gutin, P.H., Phillips, T.L., and Wilson, C.B. (1998b) Radiation therapy and hydroxyurea followed by the combination of 6-thioguanine and BCNU for the treatment of primary malignant brain tumors. Int. J. Radiat. Oncol. Biol. Phys. 40, 57-63.

Rodriguez, L.A., Prados, M., Silver, P., and Levin, V.A. (1989) Reevaluation of procarbazine for the treatment of recurrent malignant central nervous system tumors. Cancer 64, 2420-2423.

Scheibel, R.S., Meyers, C.A., and Levin, V.A. (1996) Cognitive dysfunction following surgery for intracerebral glioma: Influence of histopathology, lesion location, and treatment. J. Neurooncol. 30, 61-69.

Schold, S.C., Jr., Herndon, J.E., Burger, P.C., Halperin, E.C., Vick, N.A., Cairncross, J.G., Macdonald, D.R., Dropcho, E.J., Morawetz, R., Bigner, D.D., and Mahaley, M., Jr. (1993) Randomized comparison of diaziquone and carmustine in the treatment of adults with anaplastic glioma. J. Clin. Oncol. 11, 77-83.

Schold, S.C., Jr., Kokkinakis, D.M., Rudy, J.L., Moschel, R.C., and Pegg, A.E. (1996) Treatment of human brain tumor xenografts with O6-benzyl-2'deoxyguanosine and BCNU. Cancer Res. 56, 2076-2081.

Silvani, A., Salmaggi, A., Pozzi, A., Fariselli, L., Franzini, A., and Boiardi, A. (1995) Effectiveness of early chemotherapy treatment in anaplastic astrocytoma patients. Tumori 81, 424-428.

Van Tassel, P., Bruner, J.M., Maor, M.H., Leeds, N.E., Gleason, M.J., Yung, W.K., and Levin, V.A. (1995) MR of toxic effects of accelerated fractionation radiation therapy and carboplatin chemotherapy for malignant gliomas. Am. J. Neurorad. 16, 715-726.

Wacker, M.R., Hoshino, T., Ahn, D.K., Davis, R.L., and Prados, M.D. (1994) The prognostic implications of histologic classification and bromodeoxyuridine labeling index of mixed gliomas. J. Neurooncol. 19, 113-122.

Warnick, R.E., Prados, M.D., Mack, E.E., Chandler, K.L., Doz, F., Rabbitt, J.E., and Malec, M.K. (1994) A phase II study of intravenous carboplatin for the treatment of recurrent gliomas. J. Neurooncol. 19, 69-74.

Yung, W.K., Mechtler, L., and Gleason, M.J. (1991a) Intravenous carboplatin for recurrent malignant glioma: A phase II study. J. Clin. Oncol. 9, 860-864.

Yung, W.K., Prados, M., Levin, V.A., Fetell, M.R., Bennett, J., Mahaley, M.S., Salcman, M., and Etcubanas, E. (1991b) Intravenous recombinant interferon beta in patients with recurrent malignant gliomas: A phase I/II study. J. Clin. Oncol. 9, 1945-1949.

Yung, W.K., Janus, T.J., Maor, M., and Feun, L.G. (1992) Adjuvant chemotherapy with carmustine and cisplatin for patients with malignant gliomas. J. Neurooncol. 12, 131-135.

Yung, W.K., Levin, V.A., Kyritsis, A., Jaeckle, K., Vaickus, L., and Peterson, P. (1994) A phase II study of rlFN-beta +/- CRA in recurrent gliomas. J. Immunother. 16, 246. (Abstract)

Yung, W.K.A., Kyritsis, A.P., Gleason, M.J., and Levin, V.A. (1996) The treatment of recurrent malignant gliomas with high dose 13-cis retinoic acid. Clin. Cancer Res. 2, 1931-1935. 